\title{
La gestión de riesgo en las operaciones de bancos privados en el período 2013- 2016
}

\section{Risk management in the operations of private banks in the 2013-2016 period}

Nino Stefano Salvatore López

Christian Morán Montalvo

Sebastian Cárdenas Zambrano

Universidad de Especialidades Espíritu Santo, Ecuador

Autor para correspondencia: nsalvatore@uees.edu.ec, cmoranm@uees.edu.ec, scardenasz@uees.edu.ec

Fecha de recepción: 24 de mayo de 2018 Fecha de aceptación: 15 noviembre del 2018

Resumen: El propósito y objetivo de este trabajo es presentar un análisis teórico de los riesgos más significativos a los que se enfrenta la banca, modelos y métodos para medir la exposición al riesgo y los métodos de control, políticas y estrategias de gestión de riesgos. La metodología implementada en el presente trabajo de investigación corresponde a un enfoque mixto, en el cual se toman partes del cuantitativo y cualitativo para poder llegar a las conclusiones. Por una parte, se utilizó el método para medir el riesgo del sector bancario que se denomina como Coeficiente de Adecuación de Capital (CAP). La situación del sistema bancario ecuatoriano se encuentra en óptimo estado, la gestión del riesgo durante el periodo analizado ha sido imponderable. La política en temas financieros y bancarios ha permitido que los bancos puedan mantener las reservar necesarias, de esta manera se amortiguan los shocks ocasionados por las fluctuaciones del mercado y ciertos eventos naturales. No existen transacciones bancarias sin riesgo, por lo que es necesario garantizar un proceso de gestión de riesgos adecuado para evitar cualquier consecuencia negativa para un banco y sus activos y pasivos. El riesgo debe identificarse primero, luego medirse, regularse y administrarse de manera efectiva.

Palabras claves: riesgo operativo; gestión de riesgo; operaciones bancarias; riesgo de crédito; riesgo de liquidez

\begin{abstract}
The purpose and basic objective of this paper is to present a theoretical analysis of the most significant risks facing banks, models and methods to measure risk exposure and control methods, policies and risk management strategies. The methodology implemented in this research work corresponds to a mixed approach, in which parts of the quantitative and qualitative are taken to reach the conclusions. On the one hand, the method used to measure the risk of the banking sector was called the Capital Adequacy Coefficient (CAP). The situation of the Ecuadorian banking system is in optimum condition, risk management during the period analyzed has been imponderable. The policy on financial and banking issues has allowed banks to maintain the reserves necessary, thus cushioning the shocks caused by market fluctuations and certain natural events. There are no bank transactions without risk, so it is necessary to guarantee an adequate risk management process in a bank to avoid any negative consequences for a bank and its assets and liabilities. The risk must first be identified and then measured, regulated and managed effectively.
\end{abstract}

Key words: operational risk; risk management; banking operations; credit risk; liquidity risk 


\section{Introducción}

El tema de esta investigación son los riesgos financieros; básicos que incluyen el riesgo de liquidez y el riesgo de crédito y los riesgos de mercado que incluyen moneda, intereses, riesgo de materias primas y riesgo de capital. Los riesgos son objeto de interconexiones complejas que pueden aumentar significativamente la exposición general de los bancos al riesgo, y el impacto y la significación del riesgo en las operaciones de la banca serán analizados en el documento. Esto se relaciona principalmente con riesgo de crédito, riesgo de liquidez y riesgo de mercado (Atiénzar \& González, 2014).

El propósito y objetivo básico de este trabajo es presentar un análisis teórico de los riesgos más significativos a los que se enfrenta la banca, modelos y métodos para medir la exposición al riesgo y los métodos de control, políticas y estrategias de gestión de riesgos.

Los bancos gestionan el riesgo de crédito de toda la cartera, así como de los préstamos o transacciones individuales. La gestión eficaz del riesgo de crédito es un componente importante de un enfoque integral de gestión de riesgos y es necesario para el éxito a largo plazo de todas las organizaciones bancarias (Martínez \& \& Venegas, 2013).

Mediante el desarrollo de la política y la estrategia de crédito, así como los procedimientos para identificar, medir, supervisar y controlar el riesgo de crédito, los bancos tratan de cubrir el riesgo de crédito en todas las actividades bancarias, así como en el nivel individual de préstamos y carteras (Serrano, 2013).

Los bancos deben establecer una política y procedimientos crediticios adecuados para asegurar la implementación de la estrategia planeada y el logro del nivel de colocación deseado, es decir, el riesgo de crédito derivado de las colocaciones a individuos (Soto, 2015).

Hasta la fecha existen estudios realizados por varios autores, y reportes provenientes de la Superintendencia de Bancos relacionados al tema. Sin embargo, en su mayoría la información se encuentra desactualizada. Se pretende realizar una revisión de las diferentes teorías que aporten a la gestión del riesgo operativo dentro de las instituciones que conforman el sistema bancario privado.

Con los resultados se pretende que al entender cada uno de los riesgos, y poder estimar la exposición del sistema bancario privado y la afectación a sus operaciones. De esta manera, poder realizar recomendación relacionadas con el monitoreo, medición y control de los diferentes tipos de riesgo para que se implementen método y modelos que aplaquen y reduzcan dicha exposición.

\section{Marco Teórico}

\section{Riesgo de crédito}

El riesgo de crédito es el resultado de transacciones financieras contractuales y / o posibles entre el proveedor y el prestatario, es decir, la variación de las posibles ganancias que podrían obtenerse en una transacción financiera debido al pago tardío o incompleto del principal e intereses. El riesgo de crédito es una parte integral del negocio bancario y el riesgo más 
significativo para el negocio del banco, ya que plantea el riesgo de que el pago se posponga o finalmente no se realice, lo que, por otro lado, crea problemas en los flujos de efectivo y afecta la liquidez del banco (Carrascal, V., \& María, J. , 2017).

Los elementos de riesgo de crédito se encuentran siempre que una persona natural o jurídica toma un producto o servicio sin el pago inmediato de ese producto o servicio. El hecho es que hay una creciente oferta de crédito en el mercado. De acuerdo con su definición, el préstamo es la colocación de los activos del banco al cliente de acuerdo con los términos definidos: duración, tasa de interés, honorarios, moneda y elementos del seguro (Wilkis, 2014). Los préstamos permiten la compra de casas, automóviles, el lanzamiento de nuevos, y el desarrollo e innovación de las empresas existentes, el desarrollo de la infraestructura del gobierno local mediante la construcción de escuelas, sistemas de alcantarillado, carreteras, etc. Con respecto a los acreedores, distinguimos tres tipos de riesgo crediticio: riesgo personal o de consumo, riesgo corporativo y riesgo estatal o país (del Rio, 2015). Además de los préstamos, existen otras fuentes de riesgo crediticio dentro de las operaciones de los bancos, como la aceptación, las transacciones interbancarias, las transacciones en divisas, los contratos forward financieros, los swaps, los bonos, las acciones, la opción y la asunción de obligaciones, la concesión de garantías, entre otras (Wilkis, 2014).

El riesgo crediticio ha existido desde la creación del primer banco y, a pesar de las innovaciones en el área de los servicios financieros, es la causa única más importante de quiebras bancarias. La razón de esto es que más del $80 \%$ de los balances de los bancos en principio se relacionan con este aspecto de la gestión de riesgos (Saavedra, 2010).

Los bancos no pueden evitar por completo los riesgos crediticios, pero pueden administrarlos dentro de los límites de elegibilidad. Esto se refiere principalmente a la valoración profesional de la solvencia, la solvencia, la supervisión y el control del riesgo de crédito, así como la determinación de la existencia de un nivel adecuado de capital para cubrir dichos riesgos, que se logra mediante una política de provisión adecuada para clientes conocidos y riesgos potenciales desconocidos (Paz, 2013).

El objetivo de la gestión del riesgo de crédito es maximizar la tasa de retorno del banco ajustada al riesgo manteniendo la exposición al riesgo de crédito dentro de parámetros aceptables. Los bancos gestionan el riesgo de crédito de toda la cartera, así como los de préstamos o transacciones individuales. La gestión eficaz del riesgo de crédito es un componente importante de un enfoque integral de la gestión de riesgos y es necesaria para el éxito a largo plazo de todas las organizaciones bancarias (Martínez \& \& Venegas, 2013).

Las normas del Comité de Basilea son mecanismos para establecer la estabilidad del mercado financiero mundial y se incorporan a las Directivas de la UE para la adecuación del capital y la gestión de riesgos en actividades bancarias, regulatorias y de control. En lo que respecta a las actividades bancarias, existe un riesgo particular de riesgo de crédito en el marco del riesgo (Jeanneau \& Tovar, 2008). Con el desarrollo de la política y estrategia de crédito y los procedimientos para identificar, medir, monitorear y controlar el riesgo de crédito, el banco busca cubrir el riesgo de crédito en todas las actividades bancarias, así como a nivel de préstamo individual y nivel de cartera (Paz, 2013). 
Los bancos necesitan establecer políticas y procedimientos de crédito adecuados para garantizar la implementación de la estrategia planificada y el logro del nivel de colocación o riesgo de crédito específico que se deriva de las colocaciones a individuos (Saavedra, 2010).

Una estrategia de riesgo de crédito debe tener en cuenta los objetivos relacionados con la calidad de los préstamos, las ganancias y el crecimiento. Todos los bancos, independientemente de su tamaño, están haciendo negocios para obtener ganancias y, por lo tanto, tienen que establecer un nivel adecuado de relación riesgo y la recompensa para sus actividades, contabilizándolos en el costo del capital (del Rio, 2015).

La estrategia de los bancos debe garantizar e incluir la continuidad del acceso. Tiene que tener en cuenta varios aspectos, como los cambios y las tendencias en la economía y los cambios resultantes en la composición y calidad de toda la cartera de préstamos. Los bancos deben evaluar periódicamente la validez y complementar estas estrategias, ya que debe ser sostenible en el transcurso de una variedad de cambios económicos (Herrera \& García, 2014).

La base de la gestión del riesgo crediticio es la identificación y el análisis de los riesgos existentes y potenciales presentes en un producto o actividad específica, y esto es particularmente relevante para los nuevos productos y actividades antes de su introducción o aceptación (Carrascal, V., \& María, J. , 2017).

\section{Riesgo de liquidez}

La liquidez es el proceso de conversión no perturbada de efectivo en colocaciones de crédito y no crediticias, es decir, conversión de cuentas por cobrar en todas las bases (capital, intereses, comisiones) y en todas sus formas (crédito, valores) en efectivo con la dinámica planificada. El riesgo de liquidez del banco es significativo porque implica un riesgo que no podrá convertir indebidamente sus activos en una forma monetaria con la dinámica planificada y necesaria. La importancia del riesgo de liquidez se puede observar a través de una definición en sentido estricto y sentido más amplio (Orsikowsky, 2002).

En sentido estricto, advierte del peligro de que los valores no puedan convertirse en efectivo en el momento deseado y al precio deseado, y en un sentido más amplio, el riesgo de liquidez significa la incapacidad de cualquier forma no monetaria de activos para transformarse en un efectivo durante un período de tiempo sin pérdida. La necesidad de un banco de liquidez se puede ver dentro del marco de oferta y demanda (Soto, 2015).

Para la mayoría de los bancos, la mayor demanda de liquidez proviene del retiro de efectivo de las cuentas depositadas y la demanda de préstamos de los clientes que el banco desea conservar, ya sea en forma de nuevos créditos, señalar o retirar dinero en función de las líneas de crédito existentes (Estrada \& Morales, 2008).

El riesgo de liquidez no armonizado surge como resultado del desajuste entre activos y pasivos de los bancos. La necesidad de los bancos de grandes cantidades de activos líquidos en el futuro es el riesgo de mayor liquidez. El riesgo de liquidez del mercado surge como resultado de la menor liquidez del mercado financiero, lo que refleja la imposibilidad de vender u obtener activos líquidos (Serrano, 2013). 
El riesgo de liquidez está estrechamente relacionado con la estructura de vencimientos del balance del banco, es decir, la responsabilidad de los activos y pasivos. La relación entre el tiempo entre el depósito para un período de tiempo determinado y la colocación de fondos durante un período de tiempo específico se conoce como la estructura de vencimiento del banco. Dado que los límites de tiempo para los depósitos y colocaciones no son los mismos (generalmente la colocación es más larga que el depósito) lo que provoca la aparición de la desigualdad materna en el balance del banco (Jeanneau \& Tovar, 2008).

El riesgo de liquidez puede medirse con dos herramientas básicas: ratios de liquidez y planes de liquidez. Los cocientes de liquidez más conocidos, la relación entre préstamos y depósitos, la relación entre los activos líquidos y los pasivos totales, la relación entre los activos a corto plazo y los pasivos a corto plazo, y la relación entre los depósitos básicos y el total de los pasivos (Toro \& Palomo, 2014). En el caso de los ratios de crédito y depósitos, cuanto menor sea la proporción de préstamos y depósitos, el banco tiene una mejor posición de liquidez, mientras que es preferible exceder 1 en el ratio de activos y pasivos a corto plazo, lo que significaría que los activos a corto plazo son más altos que los pasivos a corto plazo (Guevara, 2014).

Los sistemas bancarios a nivel global poseen un plan para gestionar el riesgo de manera efectiva, mediante el cual define las formas básicas de riesgo de liquidez, fuentes de riesgo, sistema de medición de riesgos, sistema de limitación, gestión responsable de liquidez, procesos de gestión, sistema planificado de reporte de colocación, procesos de control de riesgos y definición de planes en caso de crisis de liquidez bancaria (Paz, 2013).

Al adoptar una política de liquidez claramente definida, los bancos están protegidos de posibles problemas de liquidez relacionados al comportamiento del cliente en el pasivo, pero mediante la comparación y el control del vencimiento del préstamo en activos. Los bancos deben ajustar la estructura y los parámetros de sus servicios y productos y explicar a sus clientes por qué ciertos productos tienen ciertas diferencias de tiempo (Wilkis, 2014).

\section{Riesgo operacional}

En 2003, el Comité de Basilea desarrolló las prácticas básicas para la gestión de este riesgo. Se cree que es mejor gestionar los riesgos operacionales donde aparezcan, y la planificación, la coordinación y el monitoreo deben llevarse a cabo centralmente por el departamento para la gestión de riesgos operacionales (Jiménez \& Martín, 2005). De acuerdo con el Acuerdo de Basilea, la gestión de este riesgo debe llevarse a cabo a través de cuatro pasos: identificación, evaluación, control y monitoreo (Fernández, 2007).

La evaluación del riesgo se lleva a cabo con la ayuda de técnicas cualitativas y cuantitativas. Las técnicas cualitativas incluyen: informes de casos de incidentes de pérdida, supervisión de gestión, cuestionarios para empleados, entrevistas de salida para empleados, autoevaluación de gestión y auditoría interna. Las técnicas cuantitativas se desarrollaron principalmente con el fin de determinar el costo del capital para los riesgos operacionales del banco. Los órganos reguladores desarrollaron los acuerdos de Basilea II relacionados con la adecuación del capital (González \& de la Fuente, 2014). 
Hay varios eventos que causan pérdidas, esto puede incluir errores de alineación, errores del sistema, hurto menor, quejas de clientes, etc. Las pérdidas pueden ser directas e indirectas. Existen tres modos de tratamiento del riesgo operacional. Estos son: evitación de riesgos, reducción y uso compartido y toma de riesgos (Soto, 2015).

La prevención de riesgos se practica como un tratamiento en los casos en que los márgenes de beneficio esperados de menor actividad se llevan a cabo en relación con los costos de la toma de riesgos. Tales actividades deben detenerse o ver que no sean las únicas tomadas. La decisión debe tomarse en el contexto de varios aspectos: aspecto temporal, experiencia disponible, objetivos estratégicos y riesgo reputacional (Atiénzar \& González, 2014).

El objetivo podría ser reducir la frecuencia de ocurrencia de eventos de riesgo, así como reducir la intensidad de los eventos de riesgo. Dichos instrumentos suelen ser utilizados por numerosos mecanismos organizativos y de control dentro del sistema de control interno (Rodríguez, W., Velandia, L., \& Amado, D. , 2014). El intercambio y la transferencia de riesgos son aceptables solo si el riesgo no puede reducirse adecuadamente mediante controles internos o si el control de costos es superior a las pérdidas esperadas. Otro caso en el contexto del perfil de riesgo de un banco es cuando el riesgo es demasiado grande para poder aceptarlo (Fernández, 2007).

\section{Riesgo cambiario}

El riesgo cambiario se deriva de los cambios en los tipos de cambio entre la moneda nacional de un banco en particular y otras monedas. La consecuencia del desajuste entre el valor de los activos por un lado y el capital y los pasivos denominados en moneda extranjera por el otro (o viceversa) o por el desajuste entre las deudas externas y los reclamos expresados en la moneda nacional, conlleva un riesgo cambiario. Además, la discrepancia también puede ocurrir entre el principal y el interés debidos (Venegas, Hernandez, Moreno, \& Garcia, 2017).

El riesgo cambiario es de naturaleza especulativa y, por lo tanto, puede resultar en ganancias o pérdidas, dependiendo de los cambios en el curso y de si un banco es abastecido netamente o no en una moneda extranjera. Un ejemplo de si un banco tiene una depreciación neta de la posición en moneda extranjera resultará en ganancias de un banco, y la apreciación creará una pérdida o una posición corta neta causará un efecto inverso (Sánchez \& Liset, 2015).

La consecuencia de la fluctuación en el valor de las monedas nacionales es el cambio en las tasas externas e internas que son causadas por las diferencias en la inflación. Por lo general, estas fluctuaciones están motivadas por factores macroeconómicos y duran largos periodos de tiempo (Estrada \& Morales, 2008).

Otros factores macroeconómicos que afectan a la moneda nacional son el alcance y la dirección del comercio y los flujos de capital de un país, ya que los factores a corto plazo, como los eventos políticos esperados e inesperados, las expectativas cambiantes de los participantes del mercado o el comercio de divisas basado en la especulación (Guevara, 2014).

\section{Riesgo de tasa de interés}


El riesgo de tasa de interés es uno de los riesgos más desagradables y potencialmente más riesgosos que pueden enfrentar los bancos y la consecuencia es el cambio en la tasa de interés en el mercado financiero. Lo más frecuente es que el cambio en las tasas de interés afecta directamente a la fuente más importante de ingresos bancarios y su fuente de costos más importante. También afecta el cambio en el valor de mercado de los activos y pasivos del banco, cambiando así el valor neto del banco o acciones del banco, reflejando así directamente tanto el balance como el balance del banco (González \& de la Fuente, 2014).

El valor de los bonos (valores con tasas de interés fijas) que se encuentran en las carteras de los bancos se ve particularmente influenciado, ya que a medida que aumentan las tasas de interés, el banco se enfrenta al problema de la caída del valor de mercado de los bonos, pero también al crecimiento de los ingresos (Herrera \& García, 2014).

En el caso de que caigan las tasas de interés, el banco se encuentra con el crecimiento del valor de los bonos y menores ingresos por reinversión de interés más bajo. Además del impacto de los cambios en las tasas de interés, deben tenerse en cuenta otras características de los bonos en la cartera del banco, tales como: el vencimiento del valor, la tasa de interés nominal que llevan los valores y el sistema de depreciación de valores (Orsikowsky, 2002).

\section{Metodología}

La metodología implementada en el presente trabajo de investigación corresponde a un enfoque mixto, en el cual se toman partes del cuantitativo y cualitativo para poder llegar a las conclusiones. Por una parte, se utilizó el método para medir el riesgo del sector bancario que se denomina como Coeficiente de Adecuación de Capital (CAP).

El CAP es una razón que se expresa como porcentaje para representar la exposición de los bancos por las operaciones crediticias realizadas que han sido ponderadas. La ponderación se la realiza dependiendo del nivel de riesgo que tiene el tipo de crédito, por ejemplo, un crédito para financiar un automóvil tiene más riesgo que un crédito hipotecario.

De acuerdo a lo estipulado dentro de Comité de Basilea de 1998 cuando se creó esta razón, se determinó que el nivel mínimo tiene que ser al menos el 8\% de los activos ponderados (APRs). Esta razón se define por la siguiente fórmula para su cálculo:

$$
\text { CAP }=\frac{\text { Base de Capital }}{\text { Activos Ponderados por Riesgo }}=\geq 8 \%
$$

La base de capital se define como es la sumatoria de todos los fondos propios a disposición del banco, las reservas y otros beneficios que han sido retenidos. Esto se considera todo lo que no ha distribuido entre los accionistas como parte del dividendo. Los APRs son los activos bancarios y su respectivo riesgo que se le ha sido asignada dependiendo de su seguridad.

El enfoque cualitativo que se dará en esta investigación corresponde al método deductivo mediante la revisión bibliográfica de temas relacionados con la gestión de riesgo. La información 
será recopilada de fuentes tales como la Superintendencia de Bancos y Seguros, la Asociación de Bancos Privados, y los informes semestrales o anuales emitidos por las diferentes instituciones que conforman el sistema financiero ecuatoriano.

\section{Análisis de los resultados}

Cuando analizamos el sistema bancario ecuatoriano, se analiza que el CAP ha tenido una tendencia al alta a partir del año 2012. Esto se debe principalmente a una política de crédito establecida por el gobierno anterior, en especial en créditos de consumo. Esto ha creado el capital invertido sea solvente, es decir aquel crédito que se considera como improductivo o la cartera incobrable. La situación del sistema bancario ecuatoriano se encuentra en una buena condición debido a las altas reservas que tienen estos.

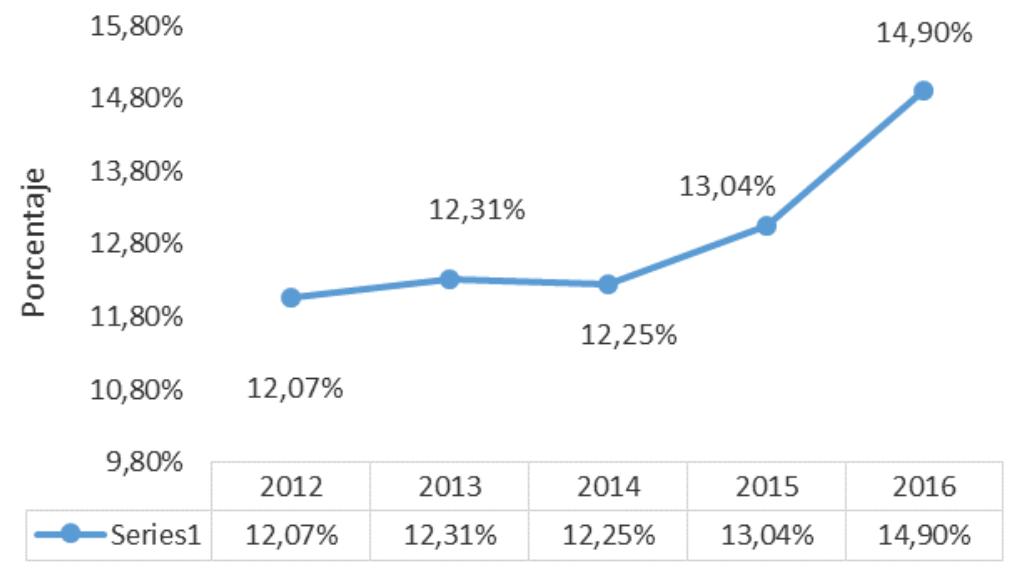

Figura 1. Evolución del CAP del sistema bancario ecuatoriano Fuente: Banco Mundial \& Banco Central del Ecuador. Elaboración propia

De acuerdo con The Economist Intelligence Unit (2017) el riesgo al que está expuesto el sector financiero ecuatoriano en relación con el resto de la región latinoamericana es más alto. Sin embargo, entre Julio 2015 y Marzo 2017, el riesgo que bordeaba el 60\% bajo cinco puntos porcentuales para situarse en 55\%. La situación del sistema bancario se considera como solido con provisiones del $117 \%$, lo cual servirá para afrontar cualquier tipo de deterioro en la cartera de crédito. Esto crea un ambiente de estabilidad que permite gestionar tipos de riesgos formulados por la reputación del sistema en conjunto.

En términos de crédito improductivo, el cual se caracteriza por ser un tipo de crédito que no genera ningún tipo de ingresos para las instituciones financieras, este ha incrementado de manera importante en este período. Se observa un incremento más acelerado especialmente a partir del año 2015, cuando se dio como terminada la bonanza petrolera. 


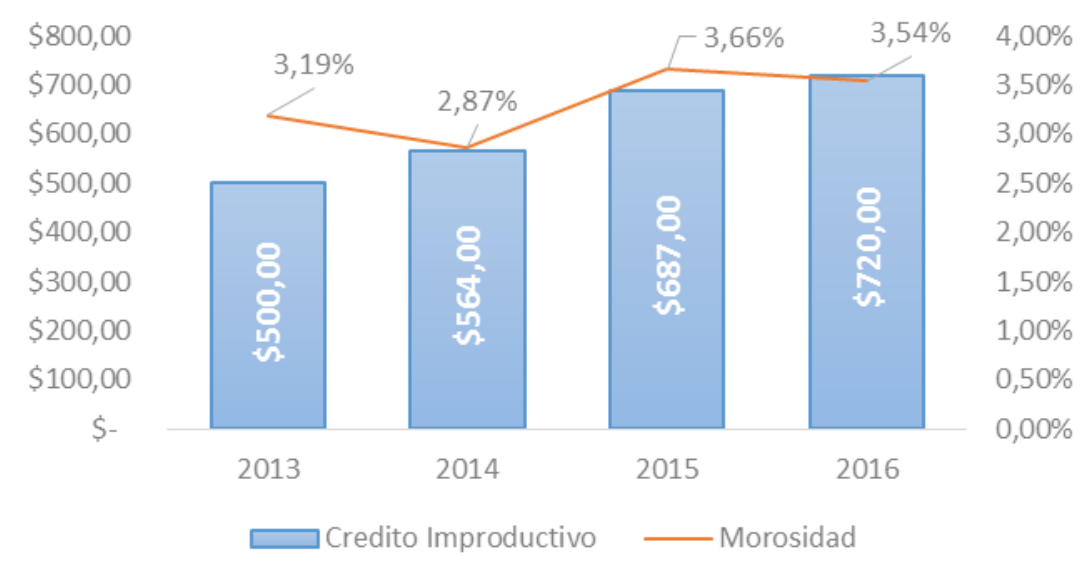

Figura 2. Evolución del crédito improductivo (en millones de dólares) y la morosidad de la cartera de crédito (\%) entre 2013 y 2016

Fuente: Asociación de Bancos Privados. Elaboración propia

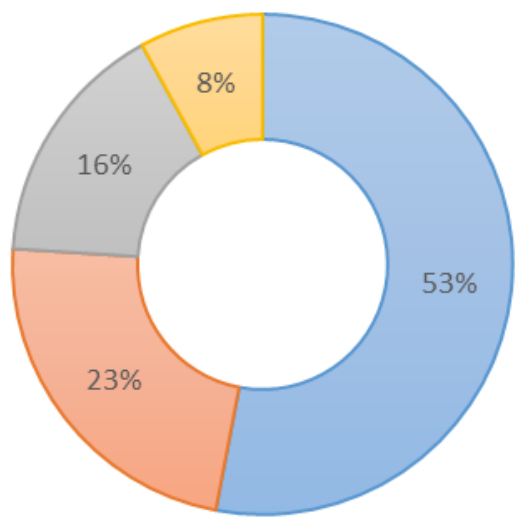

$\square$ cartera de creditos

$\square$ fondos disponibles

$\square$ inversiones

$\square$ otros

Figura 3. Distribución de activos del sistema bancario ecuatoriano

Fuente: Asociación de Bancos Privados. Elaboración propia

La diversificación de los activos del sistema financiero se ha realizado de manera adecuada, siendo la cartera de crédito la más importante, la cual representa un total de 20 mil 866 millones de dólares. Esta cartera se destina a diferentes rubros, entre los cuales se destacan el crédito comercial (50\%) y el crédito de consumo (31\%). En comparación entre el 2014 y el 2015 la variación fue de menos 870 millones de dólares, mientras que en el periodo siguiente entre 2015 y 2016 fue con un incremento de 1 mil 602 millones de dólares. 


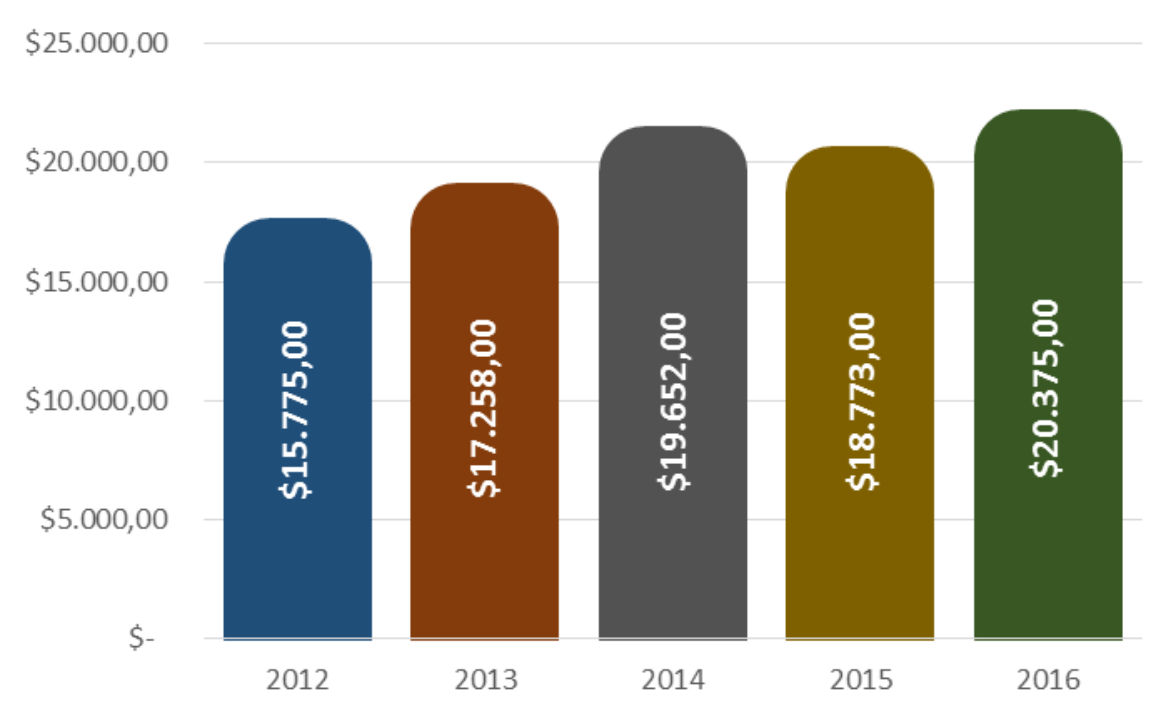

Figura 3. Evolución de la cartera de crédito entre 2012 y 2016 (en millones de dólares) Fuente: Asociación de Bancos Privados. Elaboración propia

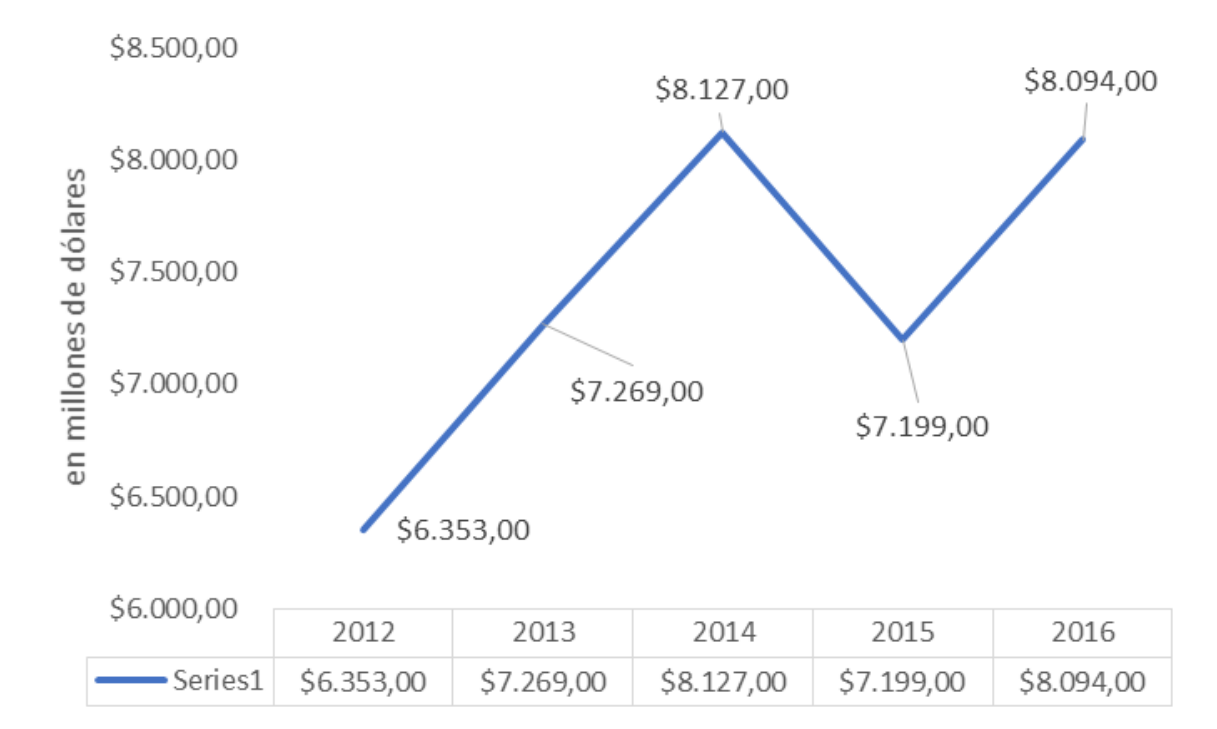

Figura 4. Evolución de los depósitos de ahorro entre 2012 y 2016 Fuente: Asociación de Bancos Privados. Elaboración propia

En la Figura 3 podemos apreciar que la cartera de crédito ha incrementado en los últimos años, sin embargo, este incremento no ha sido exagerado. Esto ha permitido que el sistema bancario ecuatoriano pueda solventar cualquier tipo de shock, tanto interno como externo. El crecimiento de la cartera de crédito no ha variado en su estructura, los créditos comerciales y de consumo siguen en los niveles superiores a los 10 y 6 mil millones de dólares anules respectivamente.

Como se puede visualizar en la Figura 4 existe una recuperación importante en los depósitos de ahorro de 2015 a 2016. El incremento se da por más de 900 millones de dólares, y eso representa que existe una confianza por parte de los usuarios del sistema bancario 
ecuatoriano, los cuales en 2015 posiblemente se vieron obligados utilizar sus fondos para solventar cualquier tipo de repercusión ocasionada por la caída de los precios del petróleo.

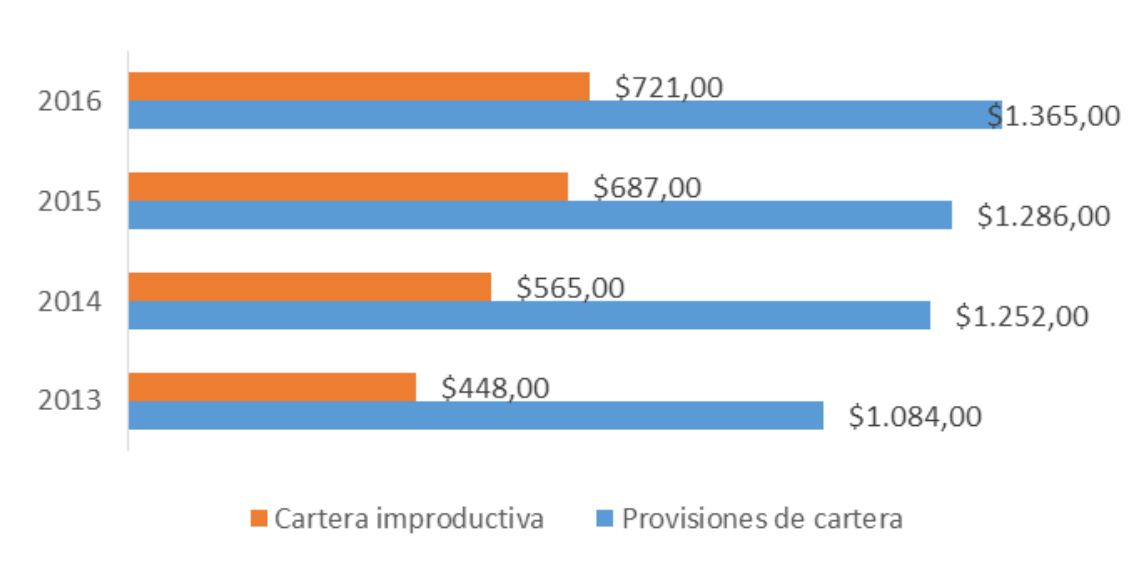

Figura 5. Cobertura de la cartera improductiva Fuente: Asociación de Bancos Privados. Elaboración propia

La cobertura de la cartera improductiva es significativa, en muchos de los casos sobrepasa del 50\% de la misma. Esto muestra cómo se puede observar en la Figura 5 una solvencia del sistema bancario ecuatoriano. En todos los aspectos que se han analizado se ha podido observar que existe un riesgo bajo, el cual ha sido gestionado mediante niveles altos de provisiones y una estabilidad en el tamaño de la cartera de crédito.

\section{Conclusiones y Recomendaciones}

La situación del sistema bancario ecuatoriano se encuentra en óptimo estado, la gestión del riesgo durante el periodo analizado ha sido imponderable. Esto se pudo visualizar dentro del índice CAP y de las estimaciones de riesgo realizadas tanto por el Banco Mundial como por The Economist Intelligence Unit. La política en temas financieros y bancarios ha permitido que los bancos puedan mantener las reservar necesarias, de esta manera se amortiguan los shocks ocasionados por las fluctuaciones del mercado y ciertos eventos naturales.

Los niveles de crédito otorgado, tanto del productivo como de consumo se han mantenido en niveles similares desde el 2013. En el 2012 se había realizado una reestructuración dentro de la legislación referente a las instituciones financieras, y se establecieron políticas que limitaban el otorgamiento de crédito (en especial de consumo). Esto tuvo los resultados deseados, y permitieron que se puedan mantener los niveles de riesgo estables, especialmente aquellos relacionados con los créditos impagables y mantuvieron constantes los niveles de depósitos.

Las medidas fueron tomadas para poder prevenir situaciones como la acontecida a finales de la década de 1990, cuando la mitad de los bancos quebraron. Las repercusiones de la liberalización del sistema financiero dados a mitad de la misma década son considerados como los precursores de esta crisis, ya que trae consigo altos riesgos. El riesgo de crédito dentro de la economía ecuatoriana es alto, debido a que la mayoría de las actividades son relacionadas a la explotación de los recursos naturales. 
Dentro de los principios económicos está indicado que los bancos siempre tomaran riesgos excesivos en sus operaciones en el caso de no ser regulados. La regulación financiera puede evitar una crisis, y eso es un evento difícil de evaluar. El aceptar el riesgo es uno de los fundamentos de las actividades de los bancos y otras instituciones financieras, lo cual se considera como una ventaja competitiva. Sin embargo debe existir un criterio coherente para establecer los parámetros de una calidad alta de crédito que es otorgado, de esta manera se puede garantizar su desenvolvimiento satisfactorio.

Con base en lo anterior, podemos concluir que no existen transacciones bancarias sin riesgo, por lo que es necesario garantizar un proceso de gestión de riesgos adecuado en un banco para evitar cualquier consecuencia negativa para un banco y sus activos y pasivos. El riesgo debe identificarse primero y luego medirse, regularse y administrarse de manera efectiva. Todo esto debe hacerse bajo la supervisión de la autoridad competente. Los bancos no pueden eliminar los riesgos por completo, pero la misión y la principal tarea de cada banco deben ser minimizarlos en la medida de lo posible.

Entre las recomendaciones que se formulan de lo investigado se puede destacar que el mantenimiento de las políticas financieras adoptadas desde hace un par de años atrás debería continuar. La liberalización del sistema financiero pudo haber tenido un riesgo operacional más elevado en la actualidad, especialmente después de la caída de los precios del petróleo en 2015. El sistema bancario ecuatoriano se encuentra en estado de solidez, debido a las provisiones y reservas que se cuentan para amortiguar los diferentes shocks.

La regularización debería expandirse con mayor énfasis en las instituciones financieras consideradas como pequeña, a pesar que estas no lleven un porcentaje alto del capital que se mueve anualmente, corresponden a sectores vulnerables dentro de la sociedad que necesita el acceso a este tipo de servicio. El riesgo de las operaciones de este tipo de instituciones tiene repercusiones en otros aspectos dentro del desarrollo de ciertos sectores de la sociedad, los cuales poseen un acceso limitado a recursos.

\section{Bibliografía}

Aguilar-Argaez, A. M., Cuadra, G., Ramírez-Bulos, C., \& Sámano, D. (2014). Anclaje de las expectativas de inflación ante choques de oferta adversos. Working Papers, Banco de México, 20-48.

Asociación de Bancos Privados del Ecuador. (2016). Evolución de la Banca. Quito: Asobanca.

Atiénzar, F. B., \& González, A. R. (2014). La gestión del riesgo operacional, un estudio en los bancos comerciales cubanos. Revista Cubana de Contabilidad y Finanzas, 99-110.

Carrascal, V., \& María, J. (2017). Modelos de medición del riesgo de crédito. Universidad Complutense de Madrid, 2-45. Del Rio, J. (2015). Riesgo de crédito. Modelización econométrica., 12-42. 
Estrada, D., \& Morales, P. (2008). La estructura del mercado interbancario y del riesgo de contagio en Colombia. Reporte de Estabilidad Financiera, 18-23.

Fernández, A. (2007). La gestión del riesgo operacional: de la teoría a su aplicación. Cantabria: Editorial Universidad de Cantabria.

González, A. R., \& de la Fuente, N. G. (2014). La gestión del riesgo operacional en el Banco de Crédito y Comercio. Revista Cubana de Contabilidad y Finanzas, 77-83.

Guevara, J. (1 de Julio de 2014). Crédito y Crecimiento Económico: El Caso de la Periferia de la Eurozona. Obtenido de http://redeconomia.org.ve/redeconomia/admin_redeconomia/uploads/investigacion/cr\%C 3\%A9dito_y_crecimiento_econ\%C3\%B3mico_en_la_eurozona.pdf

Herrera, P., \& García, J. (2014). Impacto del crédito gubernamental en el sistema financiero. . Revista Finanzas y Política Económica, 623-635.

Jeanneau, S., \& Tovar, C. (2008). Implicaciones de los mercados de bonos en moneda local para la estabilidad financiera: un resumen de los riesgos. Revista Economía Mensual, 56-65.

Jiménez, E. J., \& Martín, J. L. (2005). El nuevo acuerdo de Basilea y la gestión del riesgo operacional. Universia Business Review, 12-17.

Martínez, J. F., \& \& Venegas, F. (2013). Riesgo operacional en la banca trasnacional: un enfoque bayesiano. Ensayos-Revista de Economía, 253-269.

Orsikowsky, B. (2002). Supervisión del riesgo de liquidez. Estabilidad Financiera, 139-156. Paz, C. (2013). El riesgo de crédito en perspectiva. Madrid: Editorial UNED.

Pillai, R. (1996). Crisis and the Emergence of Charismatic Leadership in Groups: An Experimental Investigation. Journal of Applied Social Psychology,, 543-562.

Rodríguez, W., Velandia, L., \& Amado, D. . (2014). Relación entre el riesgo sistémico del sistema financiero y el sector real: un enfoque FAVAR. Ensayos sobre política económica, 1-22.

Saavedra, M. L. (2010). Modelos para medir el riesgo de crédito de la banca. Cuadernos de administración, 23-28.

Sánchez, P., \& Liset, N. (2015). ¿Qué tan conveniente es cubrir el riesgo cambiario en portafolios del MILA? Universidad de la Sabana, 23-27.

Serrano, S. C. (2013). La reestructuración del sistema bancario español tras la crisis y la solvencia de las entidades financieras: Consecuencias para las cajas de ahorros. Revista de Contabilidad, 136-146. 
Soto, F. (2015). Determinantes del riesgo de liquidez y volatilidad diferenciada de los depósitos en el sistema financiero boliviano. Desempeño de las entidades de microfinanzas ante múltiples shocks sistémicos. Revista Latinoamericana de Desarrollo Económico, 10-43.

Superintendencia de Bancos y Seguros. (30 de Diciembre de 2016). Volumen de crédito. Obtenido de http://www.sbs.gob.ec:7778/practg/sbs_index?vp_art_id=39\&vp_tip=2\&vp_buscr=41

The Economist. (17 de Marzo de 2017). Intelligence Unit. Obtenido de Ecuador: Banking sector risk: http://www.eiu.com/industry/article/395258223/ecuador-banking-sector-risk/201703-17

Toro, J., \& Palomo, R. (2014). Análisis del riesgo financiero en las PYMES-estudio de caso aplicado a la ciudad de Manizales. Revista Lasallista de Investigación, 78-88.

Venegas, F., Hernández, A., Moreno, E., \& García, A. (2017). Efectividad de las opciones installments como instrumento de cobertura ante el riesgo cambiario. Sección de Estudios de Posgrado e Investigación de la Escuela Superior de Economía del Instituto Politécnico Nacional, 59-82.

Wilkis, A. (2014). Sociología del crédito y economía de las clases populares. Revista Mexicana de Sociología, 76(2), 225-252. 